\title{
Fever and Erythema are Specific Findings in Detecting Infection Following Total Knee Arthroplasty
}

\author{
Noam Shohat ${ }^{1,2}$, Karan Goswami ${ }^{1}$,Timothy L Tan ${ }^{1}$, Brian Henstenburg1, Gabriel Makar ${ }^{1}$, Alexander J \\ Rondon ${ }^{1}$, Javad Parvizi ${ }^{\bowtie}$ \\ 1. The Rothman Institute at Thomas Jefferson University, Philadelphia, PA 19107 \\ 2. Sackler Faculty of Medicine, Tel Aviv University, Ramat Aviv, Israel \\ $\square$ Corresponding author: Javad Parvizi MD, FRCS, The Rothman Institute, 125 S 9th St. Ste 1000, Philaelphia, PA 19107. P: 267-339-7813; F: 215-503-5651; \\ Parvj@aol.com \\ (C) Ivyspring International Publisher. This is an open access article distributed under the terms of the Creative Commons Attribution (CC BY-NC) license \\ (https://creativecommons.org/licenses/by-nc/4.0/). See http://ivyspring.com/terms for full terms and conditions.
}

Received: 2018.09.20; Accepted: 2018.10.26; Published: 2019.03.16

\begin{abstract}
Current diagnostic modalities are based almost exclusively on laboratory findings and the role of clinical presentation remains unknown. The purpose of this study was to examine the diagnostic value of clinical presentation in detecting periprosthetic joint infection (PII). This study evaluated 279 patients undergoing revision surgery for failed total knee arthroplasty (TKA) between 2001-2016. Patients were classified as undergoing septic revisions based on major MSIS criteria. Aseptic revisions were defined as cases of single stage revision that did not have suspected PJI, fulfill MSIS criteria, or subsequently fail within one year of follow-up. Clinical presentation included pain, fever, presence of joint effusion or erythema, and reduced range of motion. Serum and synovial laboratory markers were also evaluated. The diagnostic value of each test was assessed and a Fagan's nomogram was constructed. A subset of MSIS-negative patients was used to demonstrate the value of various clinical presentations in detecting PJI. Post-test probability for infection was calculated taking into account clinical presentation together with serum and synovial markers. Our results show that fever and erythema are the most important signs for diagnosing PJI with a positive likelihood ratio (LR) of 10.78 and 8.08, respectively. Effusion had a LR of 2.42. Pain and reduced ROM were not as strongly correlated with PJI diagnosis; LR was 1.02 and 1.51 . Of the 35 MSIS-negative patients treated for PJI, 33 had a post-test probability of infection greater than $90 \%$ when taking clinical presentation into account. Clinical presentation should be used to guide which future diagnostic tests should be ordered and in the interpretation of their results. Our results indicate that pain, fever, presence of joint effusion or erythema, and reduced range of motion should prompt further workup for infection. We propose a nomogram that may be used in interoperating their individual weight together with laboratory findings. Fever and erythema are highly specific findings in patients with PJI and future studies should assess whether they may be added as minor criteria to current definitions for infection.
\end{abstract}

\section{Introduction}

Periprosthetic joint infection (PJI) is currently the leading cause of failure following primary total knee arthroplasty (TKA) ${ }^{1}{ }^{1}$. As the number of TKA performed each year rises, so does the number of cases presenting with PJI [2,3]. Diagnosing PJI remains a major challenge and is the first step in the management of these patients. Due to the lack of a single test that provides absolute accuracy, the diagnosis of PJI requires a combination of supportive findings. The Musculoskeletal Infection Society (MSIS) and Infectious Diseases Society of America (IDSA) have developed diagnostic criteria to standardize the definition of PJI [4,5]. These criteria are based on definite and supportive evidence for PJI, and 
have become widely accepted among surgeons worldwide [6]. While both guidelines incorporate a variety of laboratory findings, less consideration is given to clinical presentation.

Clinical presentation is evident from the first patient encounter and can be immensely helpful in assessing the pretest probability of a diagnosis, as well as the subsequent interpretation of tests ordered. This concept is applied widely across the medical profession, for instance with the calculation of a Wells pre-test probability score prior to requesting an ultrasound examination for the diagnosis of deep vein thrombosis [7]. Differences in pre-test probability may also significantly alter the post-test probability of patients with similar laboratory findings $\left[{ }^{8,9}\right]$. For instance, two patients with elevated ESR and CRP but no other elevated serum or synovial markers may have a different post-test probability for infection based on differences in their clinical presentation. While the American Academy of Orthopedic Surgeons (AAOS) acknowledge the importance of pre-test probability as evident in their stepwise approach for evaluation of patients suspected of PJI, no attention is given to bedside clinical presentation [ $\left.{ }^{10}\right]$.

Commonly reported signs or symptoms of chronic PJI may include pain, joint effusion, erythema around the joint, and fever [11-14]. They share the same biological origin as laboratory findings as they are the consequent of inflammation. While these are usually the first symptoms encountered in the evaluation of patients approaching revision arthroplasty, there is no literature, to our knowledge, that assesses the role of the physical examination for the diagnosis of PJI. The purpose of this study was to examine the diagnostic value of simple clinical findings in detecting PJI. Furthermore, we aimed to examine how the pre-test probability of clinical presentation together with current serum and synovial diagnostic markers affect the final probability of PJI.

\section{Materials and Methods}

Following Institutional Review Board approval, a single institution retrospective study evaluating all patients undergoing revision surgery for a failed TKA between January 2001 and June 2016 was performed. We included only revision TKAs that had had a minimum of two cultures (mean 4.1, range 2-6) obtained at the time of surgery and that had complete documentation of the following five clinical findings: effusion, erythema, reduced range of motion, pain, and fever. The final cohort included 279 revision TKAs, including 147 that were revised for PJI.

\section{Definition of Septic vs. Aseptic Revision:}

Patients were classified as undergoing septic

revisions if they had definitive evidence of PJI defined as major criteria by Musculoskeletal Infection Society (MSIS)[5].We excluded acute PJI defined as occurring less than 3 months from the index surgery and acute hematogenous PJI defined as acute symptoms occurring for less than 6 weeks but more than 3 month from index surgery.

Aseptic revisions were considered as those undergoing single stage revision who did not meet MSIS criteria with a diagnosis other than infection (loosening/wear, instability, malalignment, metallosis or other unexplained pain), nor had any further reoperation on the same joint within one year of follow-up. The reasons for aseptic revisions were loosening/wear (73.48\%), instability (15.90\%), malalignment $(3.78 \%)$, metalosis $(3.03 \%)$, and other unexplained pain $(3.78 \%)$. A comparison of patient characteristics and inflammatory markers between the two groups is presented in Table 1. We were interested in testing the value of clinical presentation on infected patients in whom it is difficult to reach a diagnosis of infection using serum and synovial markers. It has been previously shown that MSIS is a specific tool but lacks sensitivity with up to $30 \%$ underdiagnoses. ${ }^{15} \mathrm{We}$ were able to isolate a subgroup of patients who did not meet MSIS criteria for PJI (major or minor) but were still operated on for suspected PJI $(\mathrm{n}=81)$.Patients without serum or synovial markers available were excluded $(n=46)$. This information was used to evaluate the effect clinical presentation has on post-test probability.

Table 1. Demographics and inflammatory markers of patient undergoing septic compared to aseptic total knee revision.

\begin{tabular}{|c|c|c|c|}
\hline & $\begin{array}{l}\text { Septic Revisions } \\
(\mathrm{n}=147)\end{array}$ & $\begin{array}{l}\text { Aseptic Revisions } \\
(\mathrm{n}=132)\end{array}$ & P-value \\
\hline Age $(y r)$ & $66.98 \pm 10.54$ & $64.06 \pm 10.50$ & 0.023 \\
\hline Gender (male) & $79(53.7 \%)$ & $55(41.7 \%)$ & 0.055 \\
\hline $\mathrm{BMI}\left(\mathrm{kg} / \mathrm{m}^{2}\right)$ & $31.71 \pm 7.69$ & $32.04 \pm 5.67$ & 0.691 \\
\hline Laterality (left) & $74(50.3 \%)$ & $65(49.2 \%)$ & 0.905 \\
\hline $\begin{array}{l}\text { Primary/Revision }{ }^{\circ} \\
\text { (revision) }\end{array}$ & $54(36.73 \%)$ & $24(18.18 \%)$ & 0.001 \\
\hline $\begin{array}{l}\text { Elixhauser Comorbidity } \\
\text { Score }\end{array}$ & $2.36 \pm 1.45$ & $1.67 \pm 1.27$ & $<0.001$ \\
\hline $\operatorname{ASA}(>3)^{*}$ & $73(71.57 \%)^{*}$ & $73(55.30 \%)$ & 0.004 \\
\hline \multicolumn{4}{|l|}{ Serum markers } \\
\hline $\operatorname{ESR}(\mathrm{mm} / \mathrm{hr})$ & $69.55 \pm 32.99$ & $20.93 \pm 16.61$ & $<0.001$ \\
\hline $\mathrm{CRP}(\mathrm{mg} / \mathrm{dL})$ & $10.39 \pm 10.01$ & $0.62 \pm 0.95$ & $<0.001$ \\
\hline $\mathrm{WBC}(\mathrm{x} 103 / \mu \mathrm{L})$ & $9.30 \pm 4.14$ & $7.20 \pm 2.02$ & $<0.001$ \\
\hline PMN (\%) & $69.83 \pm 13.68$ & $60.46 \pm 7.85$ & $<0.001$ \\
\hline \multicolumn{4}{|l|}{ Synovial fluid markers } \\
\hline sWBC (cells/ $\mu \mathrm{L})$ & $90064.14 \pm 155270.56$ & $1436.13 \pm 6121.19$ & $<0.001$ \\
\hline $\operatorname{sPMN}(\%)$ & $87.07 \pm 11.69$ & $28.46 \pm 23.57$ & $<0.001$ \\
\hline \multicolumn{4}{|c|}{$\begin{array}{l}\text { Years (yr); Kilogram }(\mathrm{Kg}) \text {; Meter }(\mathrm{m}) \text {; American Society of Anesthesiologists (ASA); } \\
\text { Celsius }\left({ }^{\circ} \mathrm{C}\right) \text {; Erythrocyte Sedimentation Rate (ESR); C-Reactive Protein (CRP); } \\
\text { White Blood Cells (WBC); Polymorphonuclear Leukocytes (PMN); Synovial Fluid } \\
\text { White Blood Cells (sWBC); Synovial Fluid Polymorphonuclear Leukocytes (sPMN); } \\
\text { Millimeter (mm); Hour (hr); Milligram (mg); Microliter }(\mu \mathrm{L}) .{ }^{*} \text { Information on ASA } \\
\text { was available for } 101 \text { patients in the septic group. }{ }^{\circ} \text { Current revision is following a } \\
\text { primary or revision surgery }\end{array}$} \\
\hline
\end{tabular}




\section{Data collection}

A manual chart review of all patients meeting the above inclusion criteria was conducted from physician notes at preadmission testing. Clinical presentation included 1) presence of joint effusion as dictated by the treating surgeon, 2) presence of erythema defined as any redness of the skin surrounding the knee, 3) reduced range of motion (ROM) defined as less than 0-120 degrees, 4) patient-reported pain, and 5) fever defined as any value above $37.5^{\circ} \mathrm{C}$ on the day of admission to the hospital or subjectively reported by the patient prior to admission. Patients were included only if the above variables were explicitly stated as positive or negative by the surgeon. If any of these variables (apart from range of motion) were not specifically mentioned, the patient was excluded from the study. The follow-up visit at one year was reviewed and failure was documented as defined by the Delphi criteria [16]. Patient demographics and the Elixhauser comorbidity Index were collated from the medical record. The Elixhauser Comorbidity Index is a method of categorizing comorbidities of patients based on the International Classification of Diseases (ICD) diagnosis codes found in administrative data. The index includes 30 categories and each comorbidity category is dichotomous. Serum C-Reactive Protein (CRP) and Erythrocyte Sedimentation Rate (ESR) as well as White Blood Cells (WBC), and Polymorphonuclear Leukocytes (PMN\%) were documented. Culture results as well as Synovial Fluid White Blood Cells (sWBC), Synovial Fluid Polymorphonuclear Leukocytes (sPMN\%), and Leukocyte Esterase (LE) were also collected. MSIS cutoffs were used to stratify the different tests as normal or abnormal values [ ${ }^{5}$.

\section{Statistical Analysis}

The first step of this study was to examine the diagnostic value of simple clinical findings in detecting PJI. We calculated sensitivity and specificity, positive predictive value (PPV) and negative predictive value (NPV) for the following variables: pain, reduced range of motion, effusion, erythema, fever, ESR, CRP, ESR and CRP together, sWBC, sPMN and LE. We calculated positive and negative likelihood ratio (LR) for both clinical and laboratory markers [17]. Chi-square was used to compare between categorical variables and t-test was used when comparing continuous variables. We attempted to perform a multivariate analysis to obtain adjusted likelihood ratios however this was not feasible due to multicollinearity between variables. Since clinical presentation is usually the first diagnostic test used in clinical practice, we calculated the pre-test probability for PJI based on their PPV. We combined the pre-test probability with the likelihood ratio (LR) of current laboratory diagnostic markers to construct a Fagan's nomogram [18]. The Fagan's nomogram (Fig. 1) is a graphical tool which, in routine clinical practice, allows one to use the results of a diagnostic test to estimate a patient's probability of having disease $\left[{ }^{19}\right]$. In this nomogram, a straight line drawn from a patient's pre-test probability of disease (left axis) through the likelihood ratio of the test (middle axis) will intersect with the post-test probability of disease (right axis). We also calculated post-test odds according to the Bayes theorem by direct mathematical calculation (Post -test odds = pre-test odds $\times$ likelihood ratio) and transformed odds to probability (Post-test odds/(post-test odds +1 ) [17]. The IBMSPSS software was used for all calculations.

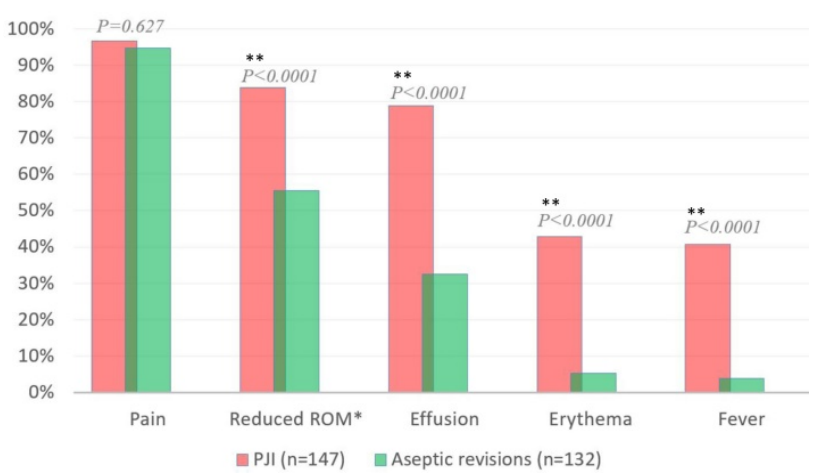

Figure 1. Clinical presentation in septic compared to aseptic revisions. Periprosthetic Join Infection (PJI); Range of Motion (ROM) * Information on range of motion was available for 175 patients (56 septic and 119 aseptic). ** Significant differences

\section{Results}

\section{Diagnostic Values for Clinical Presentation}

Fever and erythema were the most important clinical findings for diagnosing PJI; The positive likelihood ratios for fever and erythema were 10.78 (range 4.46-26.02) and 8.08 (range 3.84-17.02), respectively. While these signs were the most specific $(96.21 \%$ and $94.70 \%)$, they were also the least sensitive $(40.82 \%$ and $42.86 \%)$ for diagnosing PJI (Table 2). Sixty $(40.82 \%)$ patients undergoing septic revision complained of fever or presented with subjective fever at admission compared to only $5(3.79 \%)$ patient in the aseptic cohort $(p<0.0001)$ (Figure 1). Erythema was also significantly $(\mathrm{p}<0.0001)$ more prevalent in the septic (63 patients, $42.86 \%$ ) compared to aseptic (7 patients, $5.30 \%$ ) group.

While septic patients were more likely to suffer from effusion of the joint (116 patients, $78.91 \%$ ) compared to the aseptic patients (43 patients, $32.58 \%$ ) $(\mathrm{P}<0.001$ ), the positive likelihood ratio was 2.42 (range 
1.87-3.14). Pain and reduced ROM did not aid in the diagnosis of PJI; positive likelihood ratio was 1.02 (range 0.97-1.07) and 1.51 (1.24-1.84), respectively. Pain was the most sensitive $(96.60 \%)$ but least specific $(5.30 \%)$ complaint in both groups as it was present in $142(96.60 \%)$ septic revisions and $125(94.70 \%)$ aseptic revisions $(\mathrm{p}=0.627)$. Both groups had a reduced range of motion but this was more pronounced in the patients with aseptic revisions $(83.9 \%$ vs. $55.5 \%$; $\mathrm{p}<0.0001)$.

\section{Association between Clinical Presentation, Organism profile and Laboratory findings}

Patients undergoing septic revision who presented with fever had more comorbidities $(\mathrm{p}=0.001)$, higher serum CRP $(\mathrm{p}<0.001)$ and higher ESR ( $p=0.036)$ (Table 3). They were also two times more likely to grow resistant bacteria from the joint $(20.0 \%$ vs. $11.5 \%)$ however this did not reach statistical significance $(p=0.16)$. Interestingly, there were no

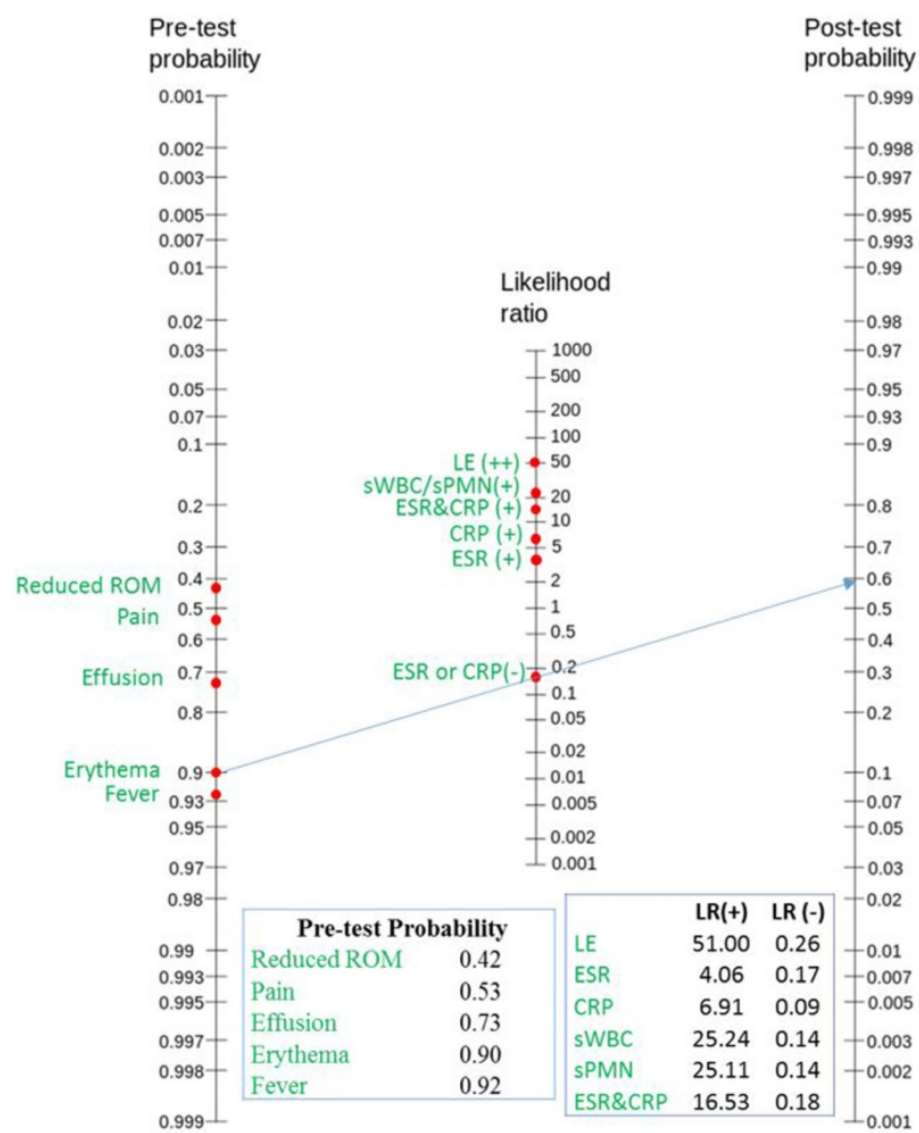

Figure 2. Fagan's nomogram taking into account the pretest probability of patients based on their clinical presentation, together with the likelihood ratio of specific serum and synovial markers. The blue arrow represents a patient with a physical examination positive for erythema. Even though his serum markers within normal levels, he has a $60 \%$ probability for infection. He went on to having a joint aspiration, which grew S.aureus on three separate cultures. Range of Motion (ROM); Erythrocyte Sedimentation Rate (ESR); C-Reactive Protein (CRP); Synovial Fluid White Blood Cells (sWBC); Synovial Fluid Polymorphonuclear Leukocytes (sPMN); Leukocyte esterase (LE); Positive Likelihood Ratio $(\mathrm{LR}+)$ = Sensitivity / (1-Specificity); Negative likelihood ratio(LR-) $=(1-$ Sensitivity $) /$ Specificity. notable differences in synovial fluid markers between patients with and without fever $(p=0.4)$. In contrast, patients with a positive effusion had significantly $(p=0.001)$ higher synovial WBC count (105461.8cells/ $\mu \mathrm{L})$ compared to patients without effusion (34072.5 cells $/ \mu \mathrm{L}$ ). Of the 147 septic revisions included in this study, 52 failed with reinfection at 1 year follow up. While there was no significant association seen between clinical findings at presentation and risk for failure $(p=1.0, p=1.0, p=0.3, p=0.4$ for pain, erythema, effusion and reduced $\mathrm{ROM}$, respectively), patient with fever did show higher failure rate $(48.1 \%$ vs. $34.8 \%$ ) and this had a tendency toward statistical significance $(\mathrm{p}=0.15)$.

\section{Probability for Infection in Clinical Practice}

A Fagan's nomogram (Figure 2) was populated. Of the 147 patients within our septic group, 23 $(15.65 \%)$ had a normal ESR or CRP. These patients may potentially be overlooked and not undergo further investigation as their likelihood ratio for PJI is low (0.18) based solely on these diagnostic tests. However, taking into account their pre-test probability based on their clinical presentation, the risk for PJI increases from $18 \%$ in a patient with only pain, to $30 \%$ in a patient with joint effusion, to $60 \%$ and $70 \%$ in a patient with erythema or fever. Notably, of the 23 patients with negative ESR or CRP, 14 had a positive effusion, 12 had joint erythema, and 4 had fever putting them at substantially higher risk for PJI.

In a subgroup analysis of 35 of patients who did not meet MSIS criteria but were still operated on for suspected PJI (Table A), considering clinical presentation resulted in an average post-test probability of $91.9 \%$ for PJI. Furthermore, 33 patients $(94.3 \%)$ had a post-test probability above $90 \%$ and twenty-five patients $(71.43 \%)$ had a post-test probability of above $95 \%$.

\section{Discussion}

Clinical presentation in PJI currently plays a limited role in established diagnostic guidelines. However, the results of our study demonstrate that fever and erythema around the joint are highly specific findings for patients with PJI. Furthermore, we reveal that clinical presentation has an important role within the diagnostic algorithm for PJI, and may substantially influence the probability of infection. These results raise the importance of adding pre-test probability to current metrics in order to diagnose infection, and further suggest that fever and erythema should receive particular attention in any future diagnostic criteria. 
Table 2. Diagnostic abilities of the various clinical presentations.

\begin{tabular}{|c|c|c|c|c|c|c|}
\hline & Sensitivity & Specificity & PPV & NPV & Positive LR & Negative LR \\
\hline Pain & $96.60 \%(92.24-98.89)$ & $5.30 \%(2.16-10.62)$ & $53.18 \%(51.92-54.44)$ & $58.33 \%(31.28-81.15)$ & $1.02(0.97-1.07)$ & $0.64(0.21-1.97)$ \\
\hline Reduced ROM & $83.93 \%(7.67-92.38)$ & $44.54 \%(35.43-53.93)$ & $41.59 \%(36.89-46.46)$ & $85.48 \%(75.80-91.72)$ & $1.51(1.24-1.84)$ & $0.36(0.19-0.68)$ \\
\hline Effusion & $78.91 \%(71.42-85.20)$ & $67.42 \%(58.73-75.32)$ & $72.96 \%(67.55-77.76)$ & $74.17 \%(67.27-80.04)$ & $2.42(1.87-3.14)$ & $0.31(0.22-0.44)$ \\
\hline Erythema & $42.86 \%(34.74-51.27)$ & $94.70 \%(89.38-97.84)$ & $90.00 \%$ (81.04-94.99) & $59.81 \%(56.26-63.26)$ & $8.08(3.84-17.02)$ & $0.60(0.52-0.70)$ \\
\hline Fever & $40.82 \%(32.79-49.22)$ & $96.21 \%(91.38-98.76)$ & $92.31 \%(83.25-96.66)$ & $59.35 \%(55.97-62.64)$ & $10.78(4.46-26.02)$ & $0.62(0.54-0.71)$ \\
\hline
\end{tabular}

Table 3._Patient characteristics and bacterial virulence stratified by clinical presentation.

\begin{tabular}{|c|c|c|c|c|c|c|c|c|c|}
\hline & \multicolumn{3}{|l|}{ Fever } & \multicolumn{3}{|l|}{ Erythema } & \multicolumn{3}{|l|}{ Effusion } \\
\hline & Yes $(n=60)$ & No $(n=87)$ & P-value & Yes $(n=63)$ & No $(n=84)$ & P-value & Yes $(n=116)$ & No $(n=31)$ & P-value \\
\hline Age $(y r)$ & 66.81 & 67.1 & 0.87 & 67.4 & 66.6 & 0.64 & 66.50 & 68.84 & 0.28 \\
\hline Gender (male) & $30(50 \%)$ & $49(62.0 \%)$ & 0.50 & $35(55.6 \%)$ & $44(52.4 \%)$ & 0.74 & $65(56.0 \%)$ & $14(45.2 \%)$ & 0.31 \\
\hline $\mathrm{BMI}\left(\mathrm{kg} / \mathrm{m}^{2}\right)$ & 31.5 & 31.9 & 0.77 & 31.1 & 32.2 & 0.42 & 31.64 & 31.99 & 0.83 \\
\hline Elixhauser & 2.83 & 2.04 & 0.001 & 2.5 & 2.2 & 0.21 & 2.37 & 2.33 & 0.91 \\
\hline S.aureus & $21(35.0 \%)$ & $24(27.6 \%)$ & 0.36 & $20(31.7 \%)$ & $25(29.8 \%)$ & 0.86 & $35(30.2 \%)$ & $10(32.3 \%)$ & 0.83 \\
\hline Resistant bacteria & $12(20.0 \%)$ & $10(11.5 \%)$ & 0.16 & $9(14.29 \%)$ & $13(15.47 \%)$ & 0.92 & $16(13.8 \%)$ & $6(19.4 \%)$ & 0.41 \\
\hline Gram (-) & $10(16.7 \%)$ & $8(9.2 \%)$ & 0.20 & $11(17.5 \%)$ & $7(8.3 \%)$ & 0.12 & $16(13.8 \%)$ & $2(6.5 \%)$ & 0.36 \\
\hline Multiple organisms & $6(6.9 \%)$ & $4(6.7 \%)$ & 1.00 & $5(7.9 \%)$ & $5(6.0 \%)$ & 0.74 & $8(6.9 \%)$ & $2(6.5 \%)$ & 1.00 \\
\hline \multicolumn{10}{|l|}{ Serum markers } \\
\hline ESR (mm/hr) & 76.4 & 64.6 & 0.036 & 67.4 & 71.1 & 0.50 & 68.1 & 75.0 & 0.32 \\
\hline CRP $(\mathrm{mg} / \mathrm{dL})$ & 15.4 & 6.8 & $<0.001$ & 10.6 & 10.2 & 0.83 & 10.7 & 8.9 & 0.41 \\
\hline \multicolumn{10}{|c|}{ Synovial fluid markers } \\
\hline sWBC (cells $/ \mu \mathrm{L})$ & 103464.8 & 78152.4 & 0.41 & 100853.1 & 80473.9 & 0.51 & 105461.8 & 34072.5 & 0.001 \\
\hline sPMN (\%) & 86.2 & 87.9 & 0.47 & 88.6 & 85.7 & 0.23 & 88.2 & 82.5 & 0.23 \\
\hline
\end{tabular}

There are two ways to interpret our results; the first is to consider clinical presentation in the pre-test probability for infection which will guide future tests and have a substantial impact upon their interpretation. Clinical presentation is available from the first patient encounter, they are non-invasive and simple. The results of this study demonstrate the immense impact they have in the evaluation the probability for infection. Current diagnostic criteria use a combination of culture and laboratory findings to define PJI [ ${ }^{4-6,20-22}$. These different criteria are all the similar in that they all reflect a snapshot of the patient state at the time they are taken, disregarding each patient's pre-test probability. Furthermore, laboratory markers may differ notably even between patients who are clinically infected [23-25]. We highlight that taking clinical presentation into account dramatically increases the probability of infection in patients who would otherwise have been diagnosed as undergoing aseptic revisions based on the current MSIS criteria. The second way to interpret and implement our results is to take these clinical preentation into consideration when assessing infection using current diagnostic criteria. While current guidelines appreciate the importance of a sinus tract and purulence, they overlook other clinical examination findings. One of the strength of the present study is the use of well-defined aseptic and septic cohorts using strict criteria. This enabled us to evaluate and score the weight of each diagnostic laboratory marker and compare them with clinical presentation.

A commonly held notion is that chronic infections present with vague symptoms such as indolent pain without systemic features. However, this has never been properly studied within the context of PJI. Published work reporting clinical presentation in PJI are retrospective cohort studies including only infected patients with PJI without a comparative aseptic cohort. Moreover, they report the findings of hips and knees, chronic and acute infections all together $[12-15,21-24]$ Similar to our findings, pain was the most frequently reported clinical manifestation in these studies, with series reporting between 79 and $100 \%$ of patients with this finding [11,13,26-29]. The very low specificity we report suggests that pain is not a very good discriminating symptom. Nevertheless, pain may be the only symptom of chronic infection (especially in cases of low virulence) and pain by itself justifies further evaluation to rule out PJI. The presence of a joint effusion has been previously reported to be significantly higher in patients with PJI compared to aseptic revisions [13]. When comparing 172 THA with 148 TKA undergoing revision surgery for PJIs, Zajons et al ${ }^{[30}$ found effusion rates of $29 \%$ and $75 \%$, 
respectively. We also showed higher rates of effusion in PJI patients compared to controls, with similar overall incidence for knee PJI. Joint stiffness and reduced ROM are underreported findings and descriptions differ widely whenever reported. Tande et al [ ${ }^{31}$ ] reported a sensitivity of $20.5 \%$ (95\% CI, 9.3 $36.5)$ and a specificity of $99.0 \%(95 \% \mathrm{CI}, 94.5-100.0)$ in a sample of 39 acute hematogenous PJI's compared with 100 non-infected controls. The incidence of reduced ROM in chronic PJI in a study by Jacobs et al reached $41.7 \%$ (25/60 patients) [ ${ }^{32}$. Tseng et al [27] found evidence of stiffness in $37.3 \%$ (22/ 59 patients). Notably these studies did not specify TKA from THA. Zajons et al [30] found an incidence of knee stiffness to be $85 \%$ (126/148 patients), similar to our findings. In our study, both effusion and reduced ROM were associated with a low likelihood ratio. These findings suggest that while they are not specific tools for diagnosing PJI, they certainly have an important role in guiding future testing and may aid in the overall diagnosis when taking into account with serum and synovial testing. Future prospective studies should examine the role of clinical presentation with more objective tools such as pain questioners, goniometer and measuring. In a population based study, evaluating 75 joint infections, Tsaras et al. [11] reported fever in 27 patients $(36.5 \%)$ and erythema in 28 patients $(38.9 \%)$. Peel and colleges [12] reported similar rates in a cohort of 163 joint infections; fever was present in $62(38 \%)$ and erythema in $68(42 \%)$. While these studies did not distinguish between acute and chronic infections, they reported findings comparable to ours. Our results suggest that fever and erythema are highly specific for detecting PJI and future studies should investigate whether they may be added as minor criteria in future diagnostic definitions.

There are several limitations to the study. Most notably, the study is retrospective and the clinical data collected relies on physician notes, lacks standardization, and thus is subjective to interprettation bias. However, the fact that the majority of patients were operated by 5 different surgeons might have helped reduce this reporting bias. Another limitation is the relative small sample size. This was due to our strict exclusion criteria and inclusion of only those patients where the clinical examination data was explicitly specified as positive or negative. While this decreased our sample size, it preserved a homogenous group and eliminated assumption bias. Finally, follow up time was relatively short for monitoring PJI (1 year) and may have influenced the lack of association between clinical presentation and failure.

Taking these limitations into account, this study takes us back to the basic skills taught in medical school, by highlighting the importance of physical examination prior to any further invasive testing. In this retrospective review we assessed the value of diagnostic approaches using clinical observation in aseptic and septic revision TKA patients to help diagnose and predict PJI. The study revealed that the most significant predictive factors for diagnosing PJI were fever and erythema. Pain, effusion and reduced range of motion were more prevalent in PJI cases and should prompt further investigation when present. We provide a framework for a better understanding of bedside clinical presentation, and how they can put diagnostic tests within an appropriate context, to guide clinicians in the process of reaching and establishing a diagnosis of PJI. Physicians should make use of the presented nomogram to assess the probability for infection, taking into account clinical presentation as a pretest probability. In cases where posttest probability is high, patients should be considered infected and treated appropriately. Future studies should assess whether fever and erythema may be added as minor criteria to current MSIS definition for infection.

Table A. Characteristics, laboratory and clinical findings of a sub-group of patients diagnosed and treated for suspected infection although not meeting MSIS criteria for infection $(n=35)$.

\begin{tabular}{ll}
\hline & MSIS (-) suspected PJI (n=35) \\
\hline Age (yr) & $62.16 \pm 10.4$ \\
Gender (male) & $17(48.57 \%)$ \\
BMI $\left(\mathrm{kg} / \mathrm{m}^{2}\right)$ & $33.27 \pm 7.2$ \\
Laterality (left) & $14(40 \%)$ \\
Primary/Revision (revision) & $9(25.7 \%)$ \\
Elixhauser Comorbidity Score & $2.14 \pm 1.3$ \\
MSIS minor criteria $*$ & $1.34 \pm 0.78$ \\
ESR (mm/hr) & $25(71.42 \%)$ \\
CRP (mg/dL) & $24(68.57 \%)$ \\
ESR\&CRP & $19(54.29 \%)$ \\
sWBC (cells/ $\mu$ L) & $15(42.86 \%)$ \\
sPMN (\%) & $9(25.71 \%)$ \\
Single positive culture & $4(11.43 \%)$ \\
LE (++) * & $4(44.44 \%)$ \\
Clinical presentation $*$ & \\
Pain & $32(91.43 \%)$ \\
Reduced ROM & $15(42.86 \%)$ \\
Effusion & $22(62.86 \%)$ \\
Erythema & $11(31.43 \%)$ \\
Fever & $6(17.14 \%)$ \\
\hline
\end{tabular}

Years (yr); Kilogram (Kg); Meter (m); Erythrocyte Sedimentation Rate (ESR); C-Reactive Protein (CRP); White Blood Cells (WBC); Polymorphonuclear Leukocytes (PMN); Synovial Fluid White Blood Cells (sWBC); Synovial Fluid Polymorphonuclear Leukocytes (sPMN); Millimeter (mm); Hour (hr); Milligram (mg); Microliter $(\mu L)$.

*Information on Leukocyte esterase (LE) was available for 9 patients. ${ }^{\circ}$ Current revision is following a primary or revision surgery. ${ }^{¥}$ Data presented as dichotomous variables (yes/no)

\section{Competing Interests}

The authors have declared that no competing interest exists. 


\section{References}

1. Bozic KJ, Kurtz SM, Lau E, et al. The epidemiology of revision total knee arthroplasty in the United States. Clin Orthop. 2010;468(1):45-51. doi:10.1007/s11999-009-0945-0

2. Kurtz S, Ong K, Lau E, Mowat F, Halpern M. Projections of primary and revision hip and knee arthroplasty in the United States from 2005 to 2030 . J Bone Joint Surg Am. 2007;89(4):780-785. doi:10.2106/JBJS.F.00222

3. Patel A, Pavlou G, Mújica-Mota RE, Toms AD. The epidemiology of revision total knee and hip arthroplasty in England and Wales: a comparative analysis with projections for the United States. A study using the National Joint Registry dataset. Bone Jt J. 2015;97-B(8):1076-1081. doi:10.1302/0301-620X.97B8. 35170

4. Osmon DR, Berbari EF, Berendt AR, et al. Executive summary: diagnosis and management of prosthetic joint infection: clinical practice guidelines by the Infectious Diseases Society of America. Clin Infect Dis Off Publ Infect Dis Soc Am. 2013;56(1):1-10. doi:10.1093/cid/cis966

5. Parvizi J, Zmistowski B, Berbari EF, et al. New Definition for Periprosthetic Joint Infection: From the Workgroup of the Musculoskeletal Infection Society. Clin Orthop. 2011;469(11):2992-2994. doi:10.1007/s11999-011-2102-9

6. Parvizi J, Gehrke T, Chen AF. Proceedings of the International Consensus on Periprosthetic Joint Infection. Bone Jt J. 2013;95-B(11):1450-1452. doi:10.1302/ 0301-620X.95B11.33135

7. Wells PS, Anderson DR, Bormanis J, et al. Value of assessment of pretest probability of deep-vein thrombosis in clinical management. Lancet Lond Engl. 1997;350(9094):1795-1798. doi:10.1016/S0140-6736(97)08140-3

8. Diamond GA, Forrester JS. Analysis of probability as an aid in the clinical diagnosis of coronary-artery disease. N Engl J Med. 1979;300(24):1350-1358. doi:10.1056/NEJM197906143002402

9. Akobeng AK. Understanding diagnostic tests 2: likelihood ratios, pre- and post-test probabilities and their use in clinical practice. Acta Paediatr Oslo Nor 1992. 2007;96(4):487-491. doi:10.1111/j.1651-2227.2006.00179.x

10. Rosemont 9400 West Higgins Road, Fax: 847.823.8125 I 60018 P 8478237186. Clinical Practice Guidelines. / guidelines/. Accessed August 7, 2017.

11. Tsaras G, Osmon DR, Mabry T, et al. Incidence, secular trends, and outcomes of prosthetic joint infection: a population-based study, olmsted county, Minnesota, 1969-2007. Infect Control Hosp Epidemiol. 2012;33(12):1207-1212. doi:10.1086/668421

12. Peel TN, Cheng AC, Buising KL, Choong PFM. Microbiological Aetiology, Epidemiology, and Clinical Profile of Prosthetic Joint Infections: Are Current Antibiotic Prophylaxis Guidelines Effective? Antimicrob Agents Chemother. 2012;56(5):2386-2391. doi:10.1128/AAC.06246-11

13. Duff GP, Lachiewicz PF, Kelley SS. Aspiration of the knee joint before revision arthroplasty. Clin Orthop. 1996;(331):132-139.

14. Sendi P, Banderet F, Graber P, Zimmerli W. Clinical comparison between exogenous and haematogenous periprosthetic joint infections caused by Staphylococcus aureus. Clin Microbiol Infect Off Publ Eur Soc Clin Microbiol Infect Dis. 2011;17(7):1098-1100. doi:10.1111/j.1469-0691.2011.03510.x

15. Parvizi J, Tan TL, Goswami K, et al. The 2018 Definition of Periprosthetic Hip and Knee Infection: An Evidence-Based and Validated Criteria. J Arthroplasty. 2018;33(5):1309-1314.e2. doi:10.1016/j.arth.2018.02.078

16. Diaz-Ledezma C, Higuera CA, Parvizi J. Success After Treatment of Periprosthetic Joint Infection: A Delphi-based International Multidisciplinary Consensus. Clin Orthop. 2013;471(7):2374-2382. doi:10.1007/s11999-013-2866-1

17. van Stralen KJ, Stel VS, Reitsma JB, Dekker FW, Zoccali C, Jager KJ. Diagnostic methods I: sensitivity, specificity, and other measures of accuracy. Kidney Int. 2009;75(12):1257-1263. doi:10.1038/ki.2009.92

18. Deeks JJ, Altman DG. Diagnostic tests 4: likelihood ratios. BMJ. 2004;329(7458): 168-169. doi:10.1136/bmj.329.7458.168

19. Fagan TJ. Letter: Nomogram for Bayes theorem. N Engl J Med. 1975;293(5):257. doi:10.1056/NEJM197507312930513

20. Riccio G, Cavagnaro L, Akkouche W, Carrega G, Felli L, Burastero G. Qualitative Alpha-defensin Versus The Main Available Tests For The Diagnosis Of Periprosthetic Joint Infection: Best Predictor Test? J Bone Jt Infect. 2018;3(3):156-164. doi:10.7150/jbji.26401

21. "Combined Diagnostic Tool" APPlication to a Retrospective Series of Patients Undergoing Total Joint Revision Surgery. https://www.ncbi.nlm.nih.gov/ pmc/articles/PMC5441141/. Accessed October 26, 2018.

22. Are There Benefits In Early Diagnosis Of Prosthetic Joint Infection With Multiplex Polymerase Chain Reaction? https:/ / www.ncbi.nlm.nih.gov/pmc/ articles/PMC5671930/. Accessed October 26, 2018.

23. Della Valle C, Parvizi J, Bauer TW, et al. Diagnosis of periprosthetic joint infections of the hip and knee. J Am Acad Orthop Surg. 2010;18(12):760-770.

24. Koh IJ, Cho W-S, Choi NY, Parvizi J, Kim TK. How accurate are orthopedic surgeons in diagnosing periprosthetic joint infection after total knee arthroplasty?: A multicenter study. The Knee. 2015;22(3):180-185. doi:10.1016/j.knee.2015.02.004

25. Limited Predictive Value of Serum Inflammatory Markers for Diagnosing Fracture-Related Infections: results of a large retrospective multicenter cohort study. https://www.ncbi.nlm.nih.gov/pmc/articles/PMC6043470/. Accessed October 26, 2018.

26. Lora-Tamayo J, Murillo O, Iribarren JA, et al. A large multicenter study of methicillin-susceptible and methicillin-resistant Staphylococcus aureus prosthetic joint infections managed with implant retention. Clin Infect Dis Off Publ Infect Dis Soc Am. 2013;56(2):182-194. doi:10.1093/cid/cis746
27. Tseng S-W, Chi C-Y, Chou C-H, et al. Eight years experience in treatment of prosthetic joint infections at a teaching hospital in Central Taiwan. J Microbiol Immunol Infect Wei Mian Yu Gan Ran Za Zhi. 2012;45(5):363-369. doi:10.1016/j.jmii.2011.12.014

28. Magnuson JE, Brown ML, Hauser MF, Berquist TH, Fitzgerald RH, Klee GG. In-111-labeled leukocyte scintigraphy in suspected orthopedic prosthesis infection: comparison with other imaging modalities. Radiology. 1988;168(1):235-239. doi:10.1148/radiology.168.1.3380966

29. Malekzadeh D, Osmon DR, Lahr BD, Hanssen AD, Berbari EF. Prior use of antimicrobial therapy is a risk factor for culture-negative prosthetic joint infection. Clin Orthop. 2010;468(8):2039-2045. doi:10.1007/s11999-010-1338-0

30. Zajonz D, Wuthe L, Rodloff AC, et al. [Infections of hip and knee endoprostheses. Spectrum of pathogens and the role of multiresistant bacteria]. Chir Z Alle Geb Oper Medizen. 2016;87(4):332-339. doi:10.1007/s00104-015-0126-5

31. Tande AJ, Palraj BR, Osmon DR, et al. Clinical Presentation, Risk Factors, and Outcomes of Hematogenous Prosthetic Joint Infection in Patients with Staphylococcus aureus Bacteremia. Am J Med. 2016;129(2):221.e11-20. doi:10.1016/j.amjmed.2015.09.006

32. The epidemiology of and risk factors for invasive Staphylococcus aureus infections in western Sweden: Scandinavian Journal of Infectious Diseases: Vol 39, No 1. https://www.tandfonline.com/doi/full/10.1080/003655406008100 26. Accessed October 26, 2018. 